Supporting Information

\title{
Sheet-Like Lignin Particles as Multifunctional Fillers in Polypropylene
}

Fenggui Chen ${ }^{1}$, Wanshuang $\mathrm{Liu}^{2}$, Seyed Ismail Seyed Shahabadi ${ }^{1}$, Jianwei Xu ${ }^{3}$, Xuehong $\mathrm{Lu}^{1, *}$

${ }^{1}$ School of Materials Science and Engineering, Nanyang Technological University, 50 Nanyang Avenue, Singapore 639798, Singapore

${ }^{2}$ Collaborative Innovation Center for Civil Aviation Composites, Donghua University, 2999 North Renmin Road, Shanghai, 201620, China

${ }^{3}$ Institute of Materials Research and Engineering, 2 Fusionopolis Way, \#08-03, Innovis, Singapore 138634, Singapore

*Corresponding author: Tel: +65 67904585

E-mail: asxhlu@ntu.edu.sg (X. Lu)

6 pages, 2 tables, and 4 figures 


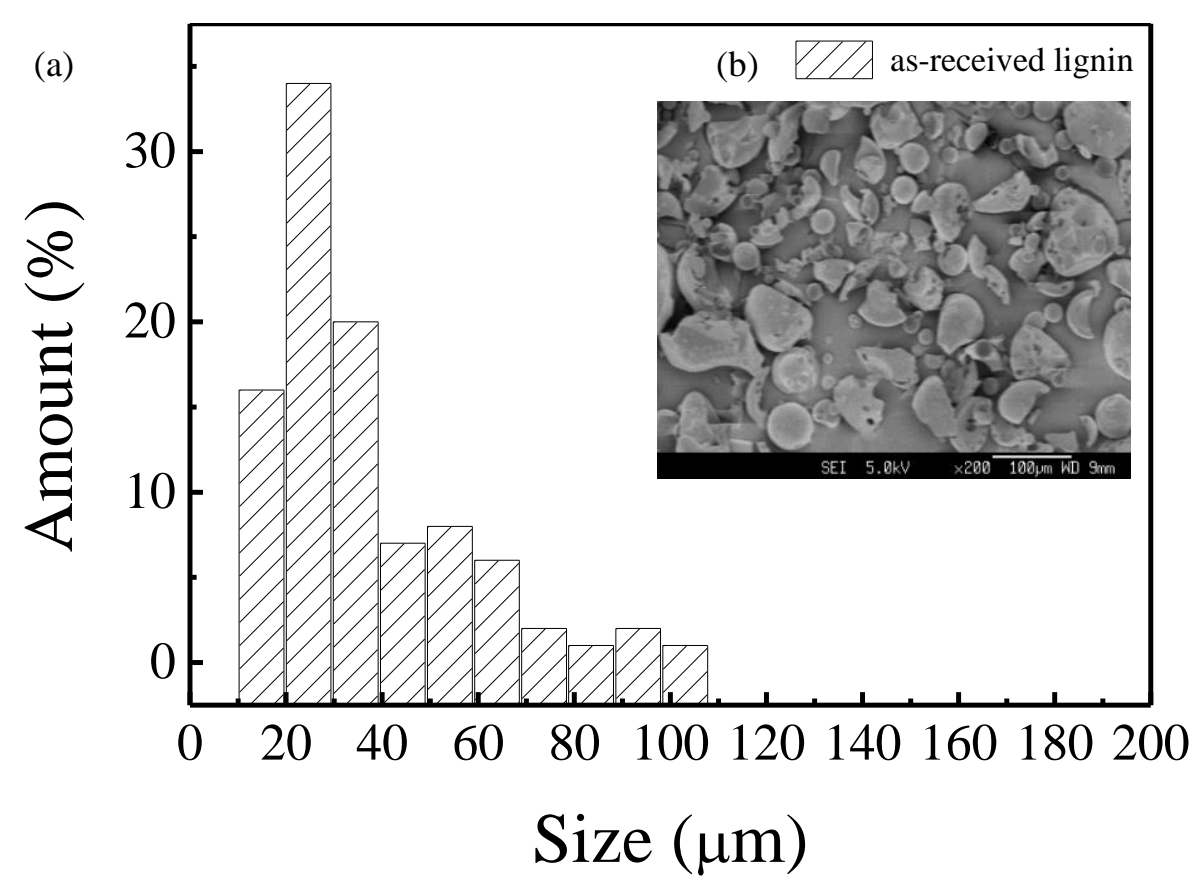

Fig. S1 Size distribution of the as-received alkali lignin measured from the SEM images $(\times 200)$ using an image analyzer.

Table S1. BET specific surface area (SSA) and BJH pore volume of lignin particles. Freeze-dried samples were prepared from $50 \mathrm{mg} / \mathrm{mL}$ lignin aqueous solutions.

\begin{tabular}{ccccc}
\hline Sample & $\begin{array}{c}\text { Freezing } \\
\text { temperature } \\
\left({ }^{\circ} \mathrm{C}\right)\end{array}$ & $\begin{array}{c}\text { Specific } \\
\text { surface area } \\
\left(\mathrm{m}^{2} / \mathrm{g}\right)\end{array}$ & $\begin{array}{c}\text { Total pore } \\
\text { volume } \\
\left(\mathrm{cm}^{3} / \mathrm{g}\right)\end{array}$ & $\begin{array}{c}\text { Average } \\
\text { pore size } \\
(\mathrm{nm})\end{array}$ \\
\hline As received lignin & - & $\sim 0.03$ & $\sim 0$ & $-^{\mathrm{a}}$ \\
Lignin-FD@50 & -196 & 9.50 & $\sim 0.01$ & $-^{\mathrm{a}}$ \\
\hline
\end{tabular}

${ }^{\mathrm{a}}$ not measured as pore volume is too small. 

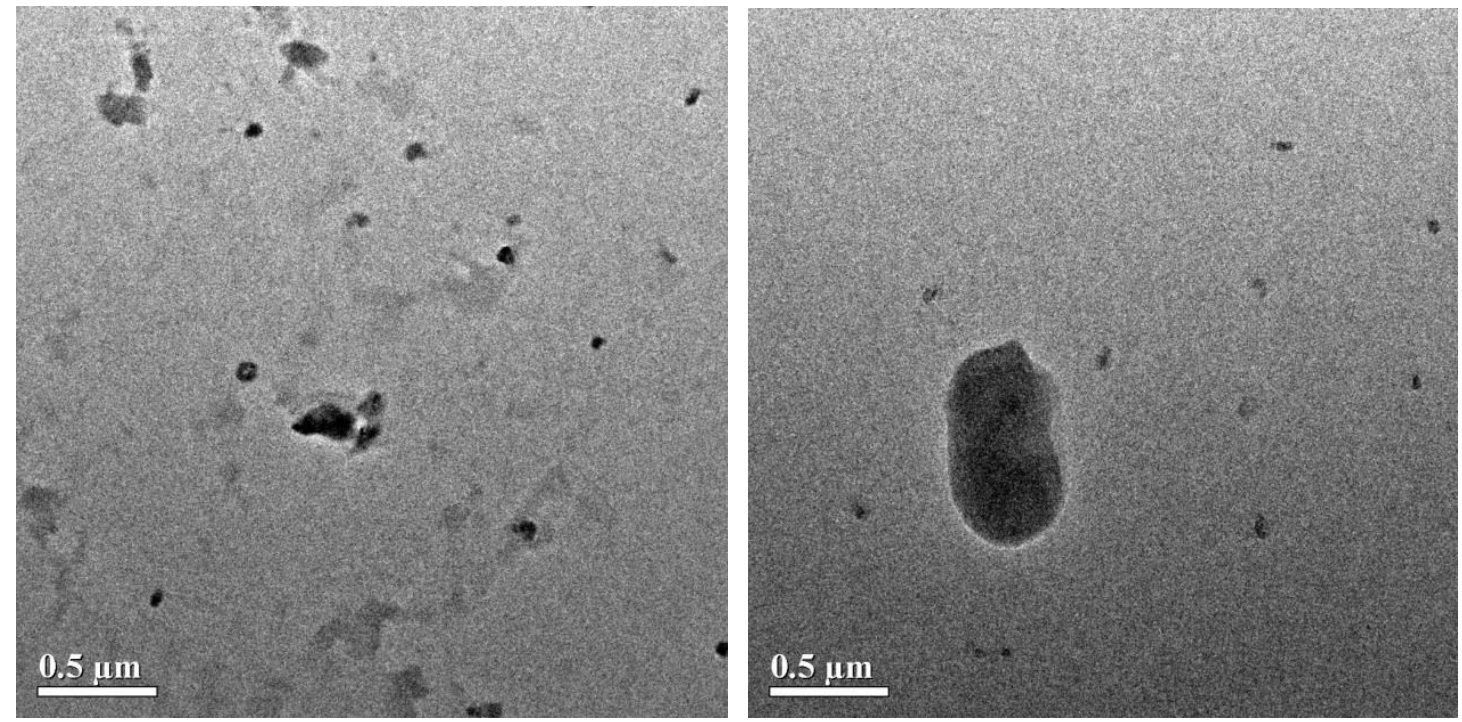

Fig. S2 Typical TEM images of PP/lignin composites containing 2 wt $\%$ freeze-dried lignin from the solution with conc. of $50 \mathrm{mg} / \mathrm{mL}$. The smaller dark particles are likely to be lignin sheets that are somewhat perpendicular to the cutting plane while the larger dark particles are likely to be lignin sheets that are more or less parallel to the cutting plane. 

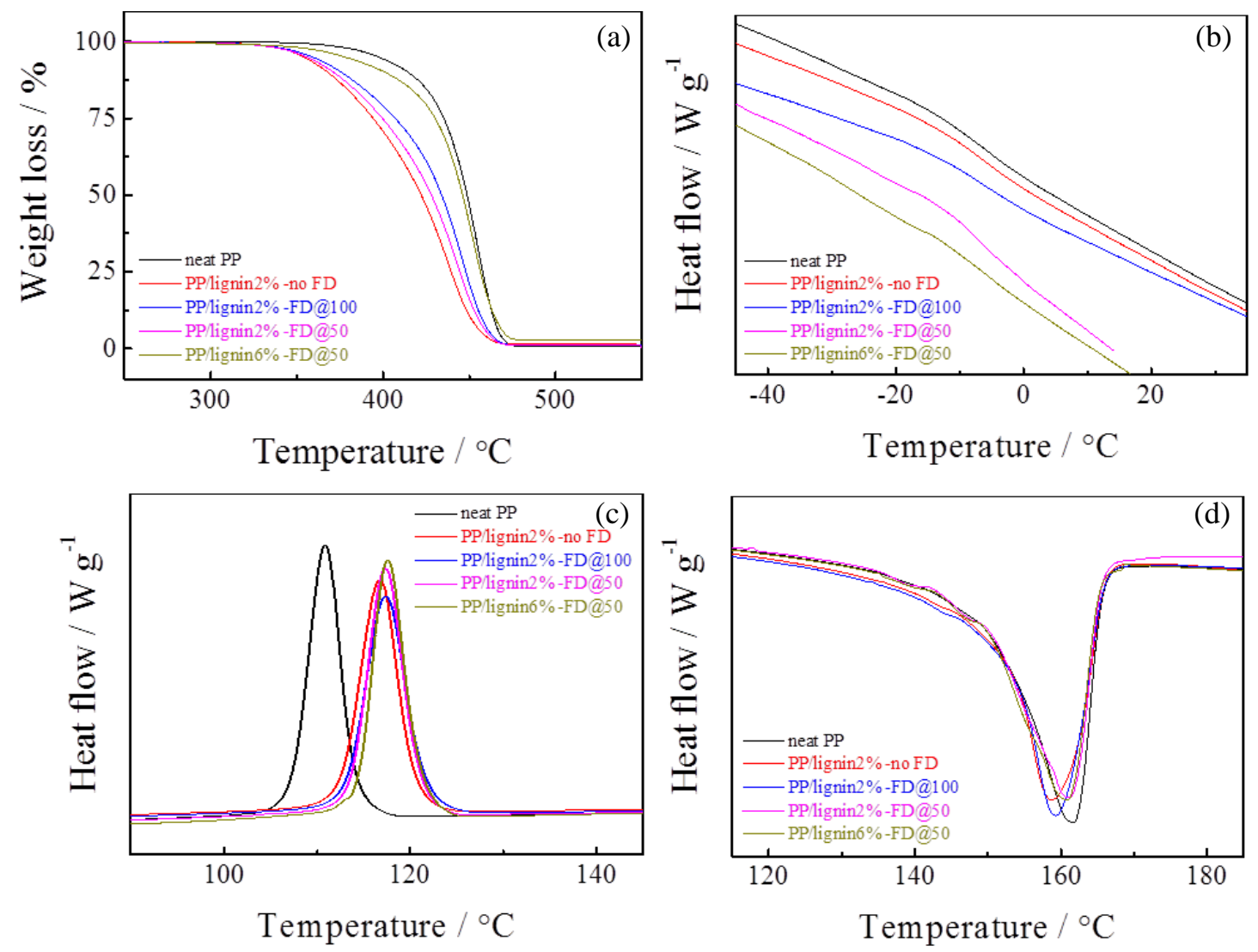

Fig. S3 Thermal properties of neat PP, and the PP/lignin composites containing 2 wt $\%$ as-received lignin, $2 \mathrm{wt} \%$ freeze-dried lignin from the solution with lignin conc. of $100 \mathrm{mg} / \mathrm{mL}$, $2 \mathrm{wt} \%$ freeze-dried lignin from the solution with conc. of $50 \mathrm{mg} / \mathrm{mL}$, and $6 \%$ freeze-dried lignin from the solution with lignin conc. of $50 \mathrm{mg} / \mathrm{mL}$. DSC was conducted at a heating rate of $10^{\circ} \mathrm{C} / \mathrm{min}$ in $\mathrm{N}_{2}$ atmosphere. 

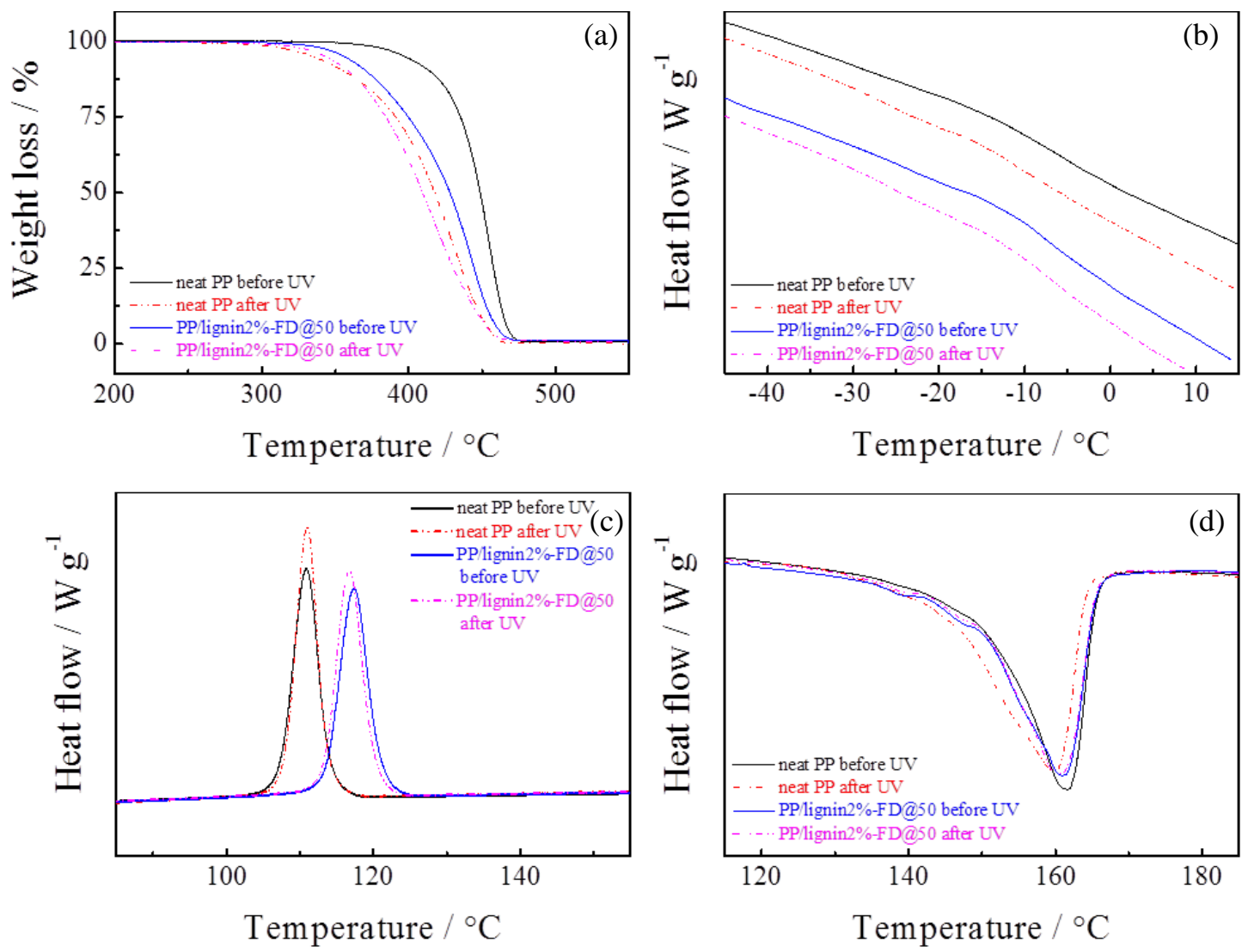

Fig. S4 Thermal properties of neat PP and PP/2\%lignin-FD@50 before and after UV irradiation. 
Table S2 Mechanical properties of neat PP and lignin blended PP composites tested with tensile speed of $50 \mathrm{~mm} / \mathrm{min}$.

\begin{tabular}{|c|c|c|c|c|c|c|c|}
\hline \multirow[b]{2}{*}{ Sample } & \multirow{2}{*}{$\begin{array}{c}\text { Lignin } \\
\text { Type } \\
\text { (mg/mL) }\end{array}$} & \multicolumn{2}{|c|}{ Composites } & \multirow{2}{*}{$\begin{array}{c}\text { E-modulus } \\
(\mathrm{MPa})\end{array}$} & \multirow{2}{*}{$\begin{array}{l}\text { Yield } \\
\text { stress } \\
(\mathrm{MPa}) \\
\end{array}$} & \multirow{2}{*}{$\begin{array}{l}\text { Yield strain } \\
(\mathrm{mm} / \mathrm{mm})\end{array}$} & \multirow{2}{*}{$\begin{array}{c}\text { Elongation } \\
\text { at break } \\
(\%)\end{array}$} \\
\hline & & $\begin{array}{c}\text { Content }^{a} \\
(w t)\end{array}$ & $\begin{array}{l}U V \\
(h)\end{array}$ & & & & \\
\hline \multirow[t]{2}{*}{ neat PP } & -- & 0 & 0 & $616 \pm 16$ & $39.1 \pm 0.8$ & $0.177 \pm 0.008$ & $54.0 \pm 5.0$ \\
\hline & -- & 0 & 336 & $650 \pm 18$ & $30.4 \pm 2.6$ & $0.065 \pm 0.006$ & $6.8 \pm 0.8$ \\
\hline \multirow[t]{2}{*}{ No FD-2\% } & no FD & $2 \%$ & 0 & $628 \pm 9$ & $38.8 \pm 0.6$ & $0.145 \pm 0.006$ & $35.3 \pm 7.2$ \\
\hline & no FD & $2 \%$ & 336 & $640 \pm 26$ & $37.5 \pm 3.3$ & $0.110 \pm 0.024$ & $12.2 \pm 3.3$ \\
\hline \multirow[t]{2}{*}{ FD@ $100-2 \%$} & 100 & $2 \%$ & 0 & $637 \pm 13$ & $38.3 \pm 0.8$ & $0.137 \pm 0.003$ & $57.8 \pm 15.5$ \\
\hline & 100 & $2 \%$ & 336 & $625 \pm 29$ & $38.7 \pm 0.7$ & $0.140 \pm 0.007$ & $48.0 \pm 11.0$ \\
\hline \multirow[t]{2}{*}{ FD@50-2\% } & 50 & $2 \%$ & 0 & $641 \pm 6$ & $38.6 \pm 1.0$ & $0.136 \pm 0.004$ & $95.5 \pm 34.9$ \\
\hline & 50 & $2 \%$ & 336 & $645 \pm 26$ & $39.6 \pm 1.0$ & $0.134 \pm 0.008$ & $68.0 \pm 18.0$ \\
\hline \multirow[t]{2}{*}{ FD@50-6\% } & 50 & $6 \%$ & 0 & $655 \pm 17$ & $37.4 \pm 1.0$ & $0.123 \pm 0.003$ & $55.0 \pm 7.3$ \\
\hline & 50 & $6 \%$ & 336 & $653 \pm 17$ & $37.9 \pm 1.1$ & $0.122 \pm 0.005$ & $38.5 \pm 7.0$ \\
\hline
\end{tabular}

\footnotetext{
${ }^{a}$ lignin content in the PP/lignin composites
} 